\title{
Dynamic Characteristic Simulation of Active Magnetic Bearings*
}

\author{
Shouichi INAMI** and Masujiro HISATANI**
}

\begin{abstract}
The step response in the axial direction of a rotor suspended by five axiscontrolled active magnetic bearings was measured and compared with two kinds of numerical simulations: the Simple model, and the FEM model. Tests were performed on a large rotor displacement which exceeded the linear range of the design condition. The agreement of the simulated results of the FEM model with those of the experi. ment was better than with those of the Simple model. Factors considered only by the FEM model were the two-dimensionality of the magnetic field including leakage flux, voltage-current characteristics of the control coil, nonlinear magnetic characteristics of the pole pieces ( $B-H$ curve), and eddy currents. The most influential factor was the magnetic leakage flux which increased the induced voltage and the time constant of the control coil.
\end{abstract}

Key Words: Vibration, Vibration Control, Response, Magnetic Bearings, Magnetic Field Analysis, FEM, Numerical Simulation, Leakage Flux

\section{Introduction}

Magnetic bearings generate no mechanical loss and need no lubricants such as oil or air as they support rotors without physical contact by controlling the magnetic attractive force. However, the spring constant of a magnetic bearing is smaller by double figures than those of rolling bearings and oil film bearings.

Against stationary external forces, including the dead weight of the rotor, the steady-state error of the rotor can be reduced to zero using an integral opera. tion of the control circuit. However, a considerably large deviation of the rotor is unavoidable against a large impulsive external force. Such external forces include those of earthquakes and the surging of com. pressors.

Contact of a high-speed rotor with electromag.

* Received 20th March, 1989. Paper No. 87-1412A

* Machinery System Research Department, Tamano Laboratory, Mitsui Engineering \& Shipbuilding CO., LTD, 16-1, Tamahara 3-chome, Tamano, Okayama, 706 Japan netic poles of the magnetic bearings or touch-down bearings should be avoided. Therefore it is important to estimate at the design stage the response of a rotor against impulsive external forces. In such a case, the rotor shows a great displacement and a large electric current occurs in the coil, the linear condition supposed at the design stage does not hold any more due to the nonlinear characteristic of the magnetic attractive force and the use of the saturated range of the $B$ $H$ curve of the magnetic poles. The effect of eddy currents also exists due to unsteadiness.

There are few reports ${ }^{(4)(5)}$ which describe the step response of a magnetic bearing system and there are no experiments or simulations for large rotor displacements.

This report shows experiments and numerical simulations concerned with step responses of a rotor supported by magnetic bearings along five axes. Electric step signals were imposed on the control circuit of the axial direction and the coil voltage, current and the rotor motion were measured. Two kinds of step signals were used with different magnitudes and directions.

The data of these experiments were compared 
with numerical simulation results. An effective method was found to estimate the rotor motion by examining the influences of the attractive force character, current character, material character and eddy current.

\section{Nomenclature}

$f:$ attractive force of magnetic pole

$I$ : current of magnetic coil

$X$ : displacement of rotor

$A$ : section area of magnetic pole $\left(5.85 \times 10^{-4} \mathrm{~m}^{2}\right)$

$N$ : number of turns (2 495 turns)

$\mu_{0}:$ magnetic permeability of vacuum

$\delta$ : clearance of thrust bearing $(1.2 \mathrm{~mm})$

$R$ : electric resistance of coil $(89 \Omega)$

$L$ : inductance of coil $\left(\mu_{0} A N^{2} / 4(\delta-X)\right)$

$M$ : rotor mass $(1 \mathrm{~kg})$

$k_{K}$ : sensitivity of displacement sensor $(1.0 \mathrm{~V} / \mathrm{mm})$

$k_{0 v}$ : amplification factor of compensation circuit network (13.2)

$T_{A}$ : time constant of phase lead compensation $(6.39$ $\left.\times 10^{-3} \mathrm{~s}\right)$

$T_{B}$ : time constant of low-pass-filter $\left(9.0 \times 10^{-4} \mathrm{~s}\right)$

$e_{b}$ : bias voltage (3.2 V) (Fig. 2)

$k_{P B}$ : power amplifier gain (5.0)

\section{Experimental Equipment}

Figure 1 shows the vertical five-axis control-type experimental equipment. Tests were performed along the controlled direction of the rotating axis (not spinning) with radial directions also controlled. A magnetic pole was working at the upper end along the axial direction, and an eddy current-type displacement sensor was placed at the lower end of the rotor.

Figure 2 shows an overall block diagram of the

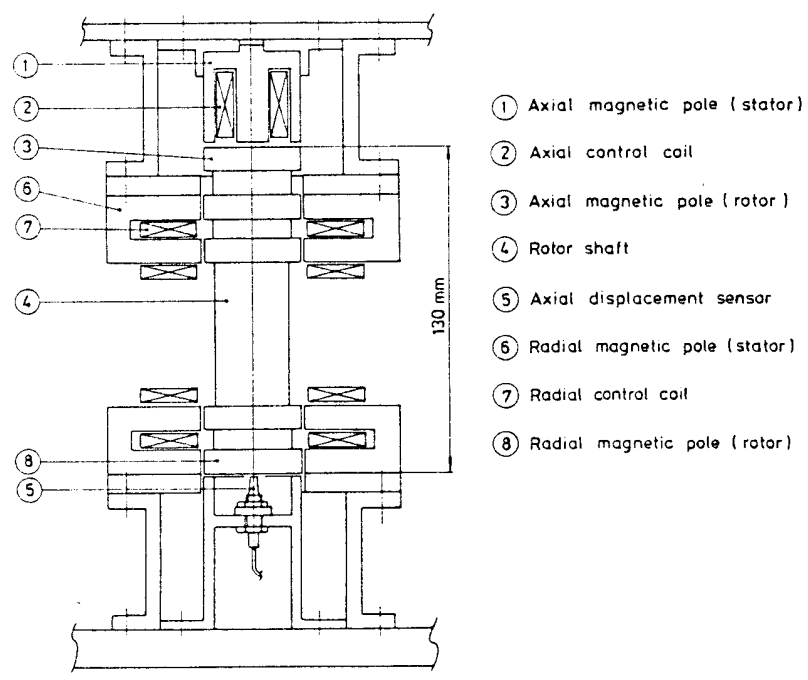

Fig. 1 Experimental equipment control system along the axial direction. A double phase-lead compensation circuit is used. $T_{A}$ is the time constant of the phase-lead compensation and $T_{B}$ is the time constant of a low-pass-filter of first-order to suppress high-frequency noise. $T_{A}$ should be set larger than $T_{B}$. The dominant eigenmodes of the system estimated at design are an oscillation mode with a frequency of $44.1 \mathrm{~Hz}$, a damping coefficient of 0.335 , and an overdamped mode with a time constant of $7.25 \mathrm{~ms}$. The static spring constant is $71.1 \mathrm{~N} / \mathrm{mm}$. The passive spring constant caused by radial bearings is more than one figure smaller.

The inputs for the power amplifier are the feedback compensation voltage $e_{f}$, the bias voltage $e_{b}$ and the pulse disturbance voltage $e_{p}$. The output is the control coil voltage $e_{c}$.

The disturbance voltage $e_{p}$ is a one-shot pulse generated by a monostable multivibrator and a mechanical switch. The width of $e_{p}$ can be varied between $60 \sim 120 \mathrm{~ms}$, the height between $\pm 13 \mathrm{~V}$.

\section{Experimental Results}

The following two experiments were performed. At the equilibrium point, the air gap " $\delta$ " between the rotor and the stator in the axial direction was $1.2 \mathrm{~mm}$.

(1) "Small displacement experiment" $e_{p}=+3.5 \mathrm{~V}$ was given. The rotor moved downward with a maximum displacement of about $0.3 \mathrm{~mm}$. (Fig. 3, a solid line)

(2) "Large displacement experiment" $e_{p}=-5.9 \mathrm{~V}$ was given. The rotor moved upward with a maximum displacement of about $0.8 \mathrm{~mm}$. (Fig.4, a solid line) The voltage $e_{c}$, the current $I$ and the displacement of the rotor $X$ were digitally recorded at intervals of $0.2 \mathrm{~ms}$ after $e_{p}$ was given.

In Fig. 3, $e_{c}$ drops to zero due to $e_{p}$ rising, but it does not become negative due to the structure of the circuit. Corresponding to the motion of the rotor, the compensation circuit adjusts output voltage $e_{c}$, and the whole system virtually reaches a new equilibrium point after $60 \mathrm{~ms}$. As the compensation circuit greatly

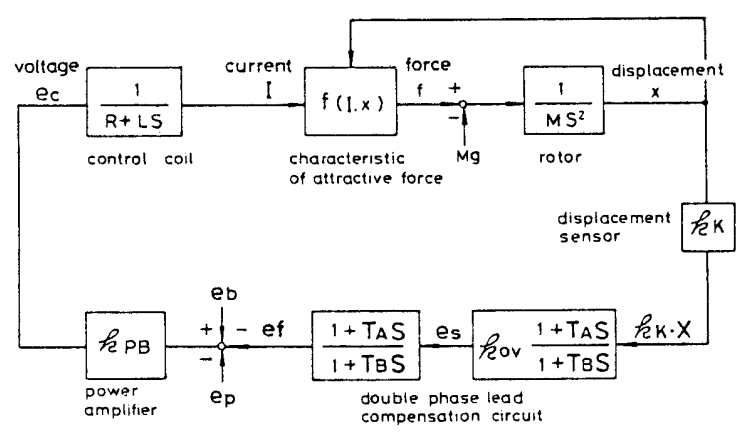

Fig. 2 Overall block diagram 
amplifies high frequency signals, $e_{c}$ contains much noise. Current $I$ and displacement $X$ have smaller noise than $e_{c}$ and change more moderately. This indicates that the coil and rotor function as the first- and the second-order low-pass-filter, respectively.

In Fig. $4, e_{c}$ reaches the power source voltage of $45 \mathrm{~V}$ due to the sharp drop of $e_{p}$. Conversely, $e_{c}$ later falls to zero with the lift of the rotor, and the control

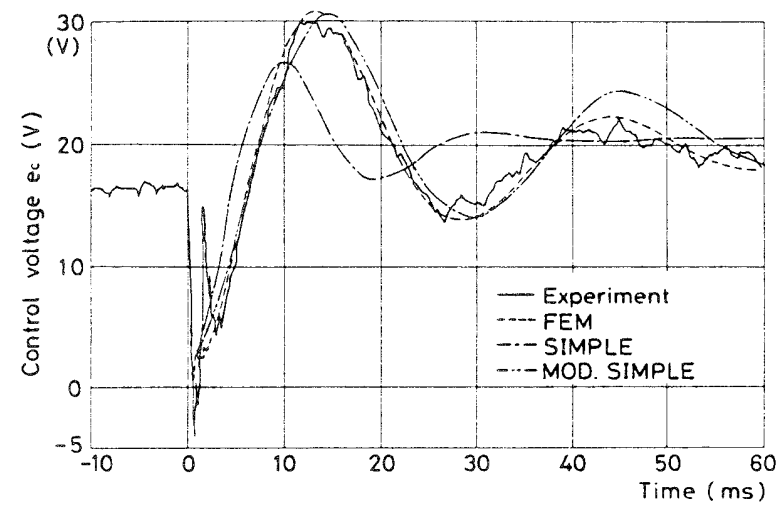

(a) Control voltage

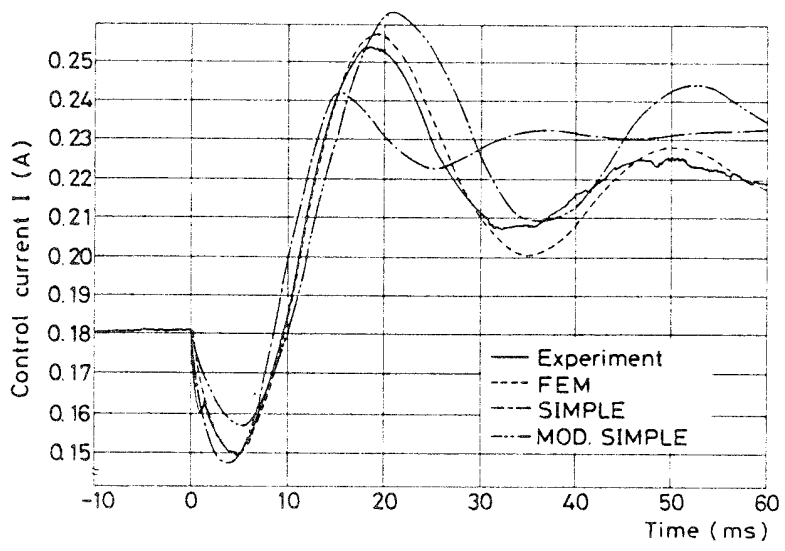

(b) Control current

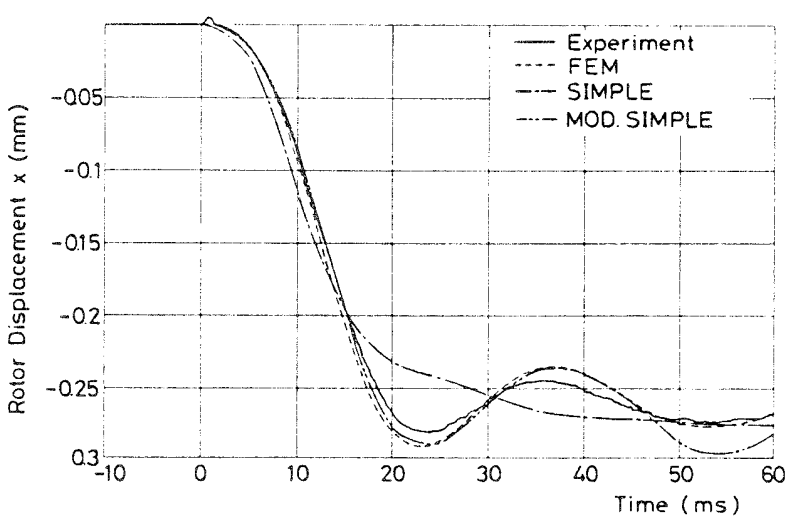

(c) Rotor displacement

Fig. 3 Response of the small downward displacement experiment coil is "switched off". when the rotor moves significantly up to $70 \%$ of the air gap, the control circuit temporarily performs an on-off control. This action is obviously a nonlinear control and deviates largely from the linear design condition. This is the primary factor which necessitates numerical calculation at the design stage to inspect stability for a large displacement of the rotor in addition to the attractive

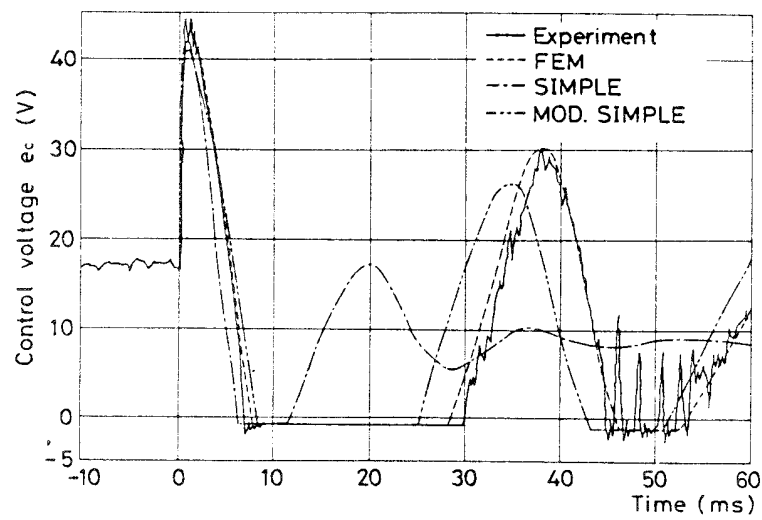

(a) Control voltage

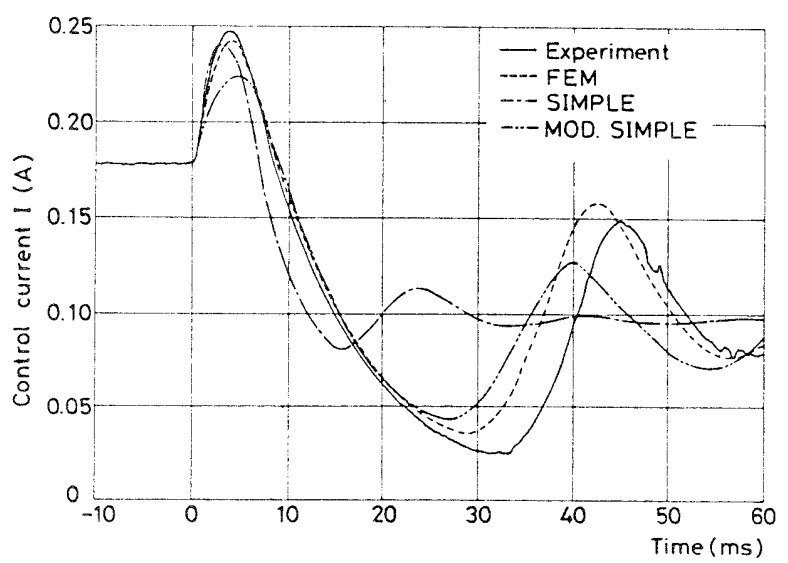

(b) Control current

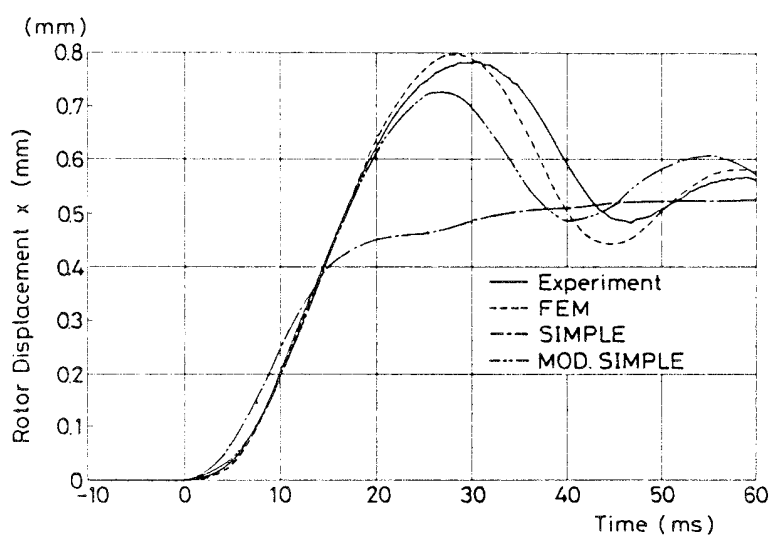

(c) Rotor displacement

Fig. 4 Response of the large upward displacement experiment 
character of the magnet and nonlinearity of the material character stated later.

\section{Numerical Simulation}

Three models, the Simple model, FEM model and Modified Simple model, were used for numerlcal slmulations.

\section{1 Simple model}

Supposing a one-dimensional magnetic circuit with no magnetic leakage flux, the following equations are obtained.

$$
\begin{aligned}
& f=M \ddot{X}+M g=\left(\frac{I}{\delta-X}\right)^{2} \times\left(\frac{\delta R}{k_{P B} e_{b}}\right)^{2} M g \\
& e_{c}=k_{P B}\left(e_{b}-e_{f}-e_{p}\right)=R I+\frac{d(L I)}{d t} \\
& k_{0 \nu} k_{K}\left(X+T_{A} \frac{d x}{d t}\right)=e_{s}+T_{B} \frac{d e_{s}}{d t} \\
& e_{s}+T_{A} \frac{d e_{s}}{d t}=e_{f}+T_{B} \frac{d e_{f}}{d t}
\end{aligned}
$$

Here $L$ is treated as a function of $X$ and the magnetic permeability of the material constant. The trapezoidal rule was used for integration with the time interval $\Delta t=0.01 \mathrm{~ms}$.

The calculation results are shown in Figs. 3 and 4 by alternate long-and-short-dashed lines. They are very different from experimental results, even qualitatively. The oscillation of displacement $X$ appears much more damped than in reality. In the static magnetic field, the attractive force character expressed by Eq. (1) corresponds with that of results within a few percent of relative error, but it is difficult to estimate the absolute value of the attractive force by the Simple model because of the existence of magnetic leakage flux.

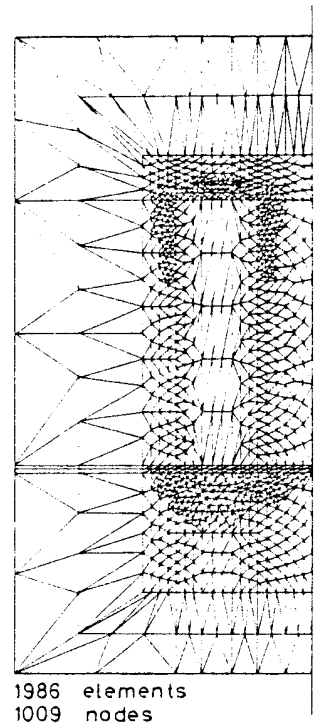

Fig. 5 Flowchart and finite elements model
Equation(1) shows the nonlinearity of the attractive force which is proportional to (current/air gap $)^{2}$. If the attractive force is linearized around the equilibrium point with respect to current and displacement, the consequence is similar. This indicates that the cause of the difference from experimental values is not the attractive force character or its nonlinearity.

\section{2 Finite Element Method model}

The FEM of the axially symmetric case was used with the magnetic vector potential. The attractive force was calculated using the simulated magnetic flux distribution by the Maxwell stress method.

Figure 5 shows the flowchart and the finite element model for the simulation. The magnetic pole material is soft magnetic iron and the measured magnetization curve is shown in Fig.6.

Calculation results are shown in Figs. 3 and 4 by broken lines. The FEM simulates the experiments very'well to within a few percent of relative error, not only for "small displacement" , but also for "large

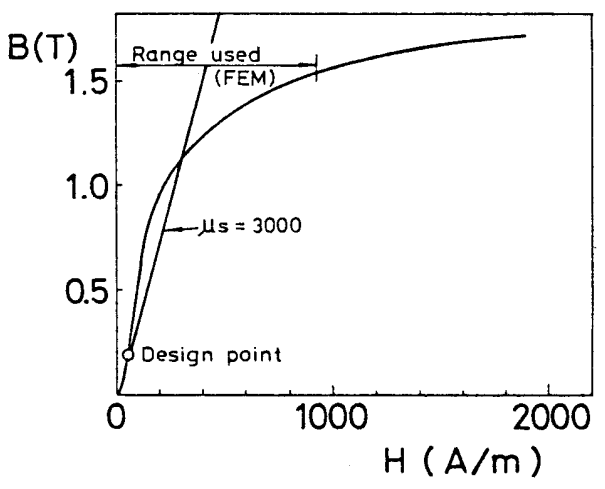

Fig. 6 Magnetization curve of soft magnetic iron

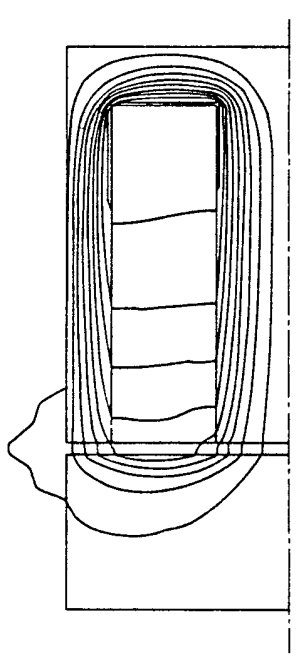

(a) Equilibrium state

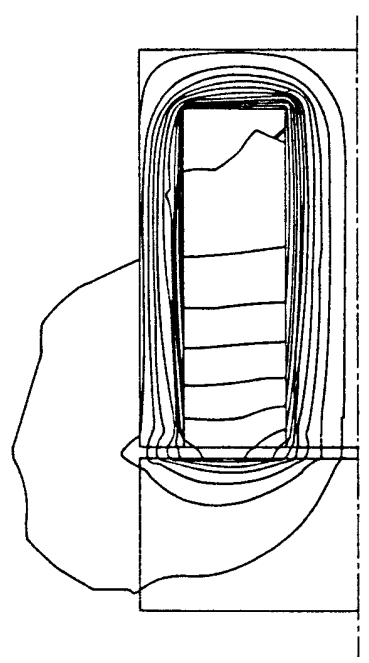

(b) After $20 \mathrm{~ms}$ of the "large displacement experiment"
Fig. 7 Contours of magnetic flux 
displacement".

Figure 7 shows contours of magnetic flux at equilibrium (a) and after $20 \mathrm{~ms}$ ( b ) of the "large displacement experiment". In Fig. 7(b), magnetic flux density has increased and magnetic flux is locally concentrated on account of the increase of the current. Magnetlc flux, which does not contribute to the attractive force, exists to a considerable extent in both cases.

Differences between the FEM model and Simple model can be found in :

(1) the calculation of the attractive force taking into consideration a two-dimensional magnetic field:

(2) the current character with the transition of the magnetic field (the second term of the right-hand side of Eq. (2));

(3) the consideration of the magnetization curve of the magnetic pole material;and

(4) the consideration of eddy current

As for (1) the FEM model is not much different from the Simple model if the absolute value of the attractive force is matched at equilibrium as stated before. A calculation with a constant magnetic permeability brought a similar result. This indicates that the influence of the material character of (3) is small because the magnetic resistance of the air gap is much larger than that of the magnetic pole. The calculations ( 4 ) of three different electric conductivities for the "large displacement experiment" are shown in Fig. 8. It can be seen that eddy current works as a resistance against the change of magnetic field and delays the response, but this is negligible. Electric conductivity is $7.0 \times 10^{6}(\Omega \cdot \mathrm{m})^{-1}$ for the calculations of Figs. 3 and 4 . The influence of eddy current is small on axial bearings, but might be large on radial bearings supporting high-speed rotors.

The above shows that the difference between the Simple model and the FEM model is in the current character of (2), namely, the increase of the coil-

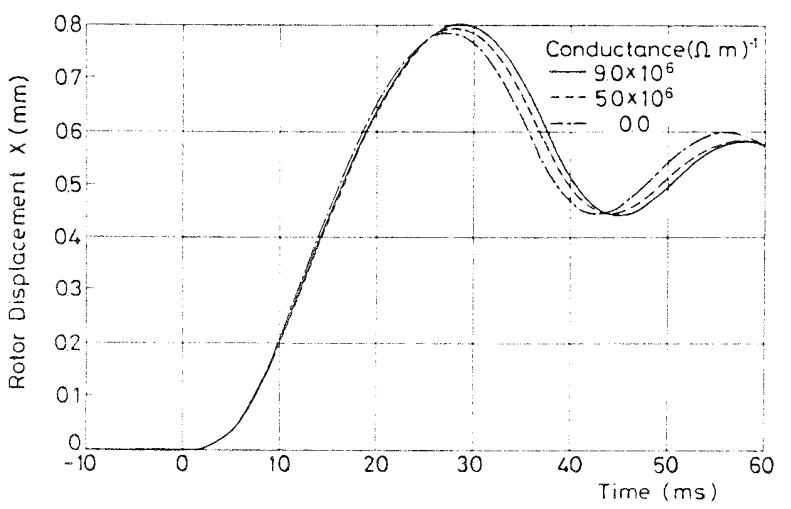

Fig. 8 Calculation results of different electric conductiv. ities induced voltage by a magnetic flux which does not contribute to the attractive force.

\section{3 Modified Simple model}

Simulation by the FEM model agrees well with experiments if the mesh division is reasonable, but it takes much effort and calculation. Therefore, the Simple model is modified as follows. As contours of magnetic flux shown in Fig. 7 contain much magnetic leakage flux which does not contribute to the attractive force, the second term of the right-hand side of Eq. (2), referring to Fig. 9, is described as follows for the FEM model and Simple model, respectively:

$$
\begin{aligned}
& V_{s}=N \frac{d \phi_{0}}{d t}\left(=\frac{d(L I)}{d t}\right) \\
& \text { FEM } V_{F}=N \frac{d \phi_{0}}{d t}+\sum_{i} N_{i} \frac{d \phi_{i}}{d t}
\end{aligned}
$$

where $N$ is the number of turns of the coil, $\phi_{o}$ the magnetic flux passing through the air gap, and $\phi_{i}$, the magnetic leakage flux taking a magnetic shortcut of number $i$ generated by $N_{i}$ turns.

Magnetic resistance $R_{m i}$ for the shortcut can be expressed as follows:

$$
\left\{\begin{array}{l}
\phi_{i}=N_{i} I / R_{m i} \\
R_{m i} \simeq \frac{\ln \left(r_{2} / r_{1}\right)}{2 \pi \mu_{0} d z} \\
N i=N \cdot Z / h
\end{array}\right.
$$

From Eq. $(5 \cdot b)$, we can obtain the modification factor by replacing the summation symbol $\Sigma$ with an integral symbol.

$$
\frac{V_{F}}{V_{S}}=1+\frac{2 \pi \mu_{1} h R_{m 0}}{3 \ln \left(r_{2} / r_{1}\right)}
$$

where $R_{m 0}$ is the magnetic resistance of the air gap and $\mu_{0}$ is the magnetic permeability of a vacuum. The second term on the right-hand side is the ratio of the magnetic resistance of the magnetic shotcut to $R_{m 0}$. The taller the coil and the smaller the ratio of $r_{2} / r_{1}$ are, the more shortcut flux there is and the larger the modification factor becomes.

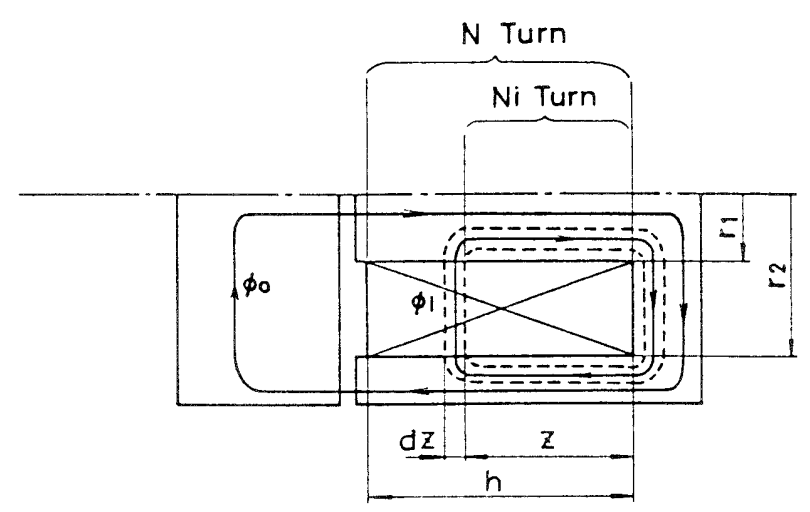

Fig. 9 Magnetic shortcut model 
The alternate long-and two-short-dashed lines in Figs. 3 and 4 show calculation results after multiplying the second term of the right-hand side of Eq. (2) by the modification factor. The difference of these results from experimental values is a little larger than that of the FEM model but much smaller than that of the Simple model. The magnetic shortcut flux increases the coil-induced voltage and the time constant of the coil. Consequently, the damping coefficient of the system eigenvalue decreases.

Thus, with the use of the FEM model and the Modified Simple model, good estimation of the response of a rotor against an impulsive force was obtained. But there still existed a small difference from the experimental value. This can be considered to be caused by the hysteresis of the magnetic material or magnetic viscosity. The response of flux density at the center of the air gap against the coil electric current was measured using a Hall generator, and a frequency characteristic was obtained which resembled the first-order lag element. Consideration of these factors is necessary for a stricter simulation, but the modified Simple model will be enough to estimate impact response at the design stage. Apply. ing the FEM model to radial bearings will occupy greater capacity and more computer time for the simulation because of the three-dimensionallty. It is easier to apply the Modified Simple model.

\section{Conclusions}

The following results were found in this study:

(1) The step response of a magnetic bearing system against an impulsive external force can be simulated with good accuracy by FEM magnetic field analysis. Even when the rotor travels as much as $70 \%$ of the air gap, the calculation agrees with the experimental value within an error a few percent.

(2) Simulation by the Simple model shows a response more damped than the experiment. This is because the Simple model neglects magnetic leakage flux through a shortcut which does not contribute to the attractive force. This leakage flux increases the induced voltage and the time constant of the control coil and decreases the damping coefficient of the system eigenvalue. A modified Simple model considering magnetic leakage flux through a shortcut generates better results.

(3) For the axial magnetic bearing with a stationary rotor, nonlinearity and the two-dimensional character of the attractive force, and the magnetic character of the pole material and generation of eddy current do not signicantly affect the dynamic characteristics.

\section{Acknowledgement}

The authors are greatly indebted to Professor Nakata and Assistant Professor Takahashi of the Department of Electrical Engineering at Okayama University for the FEM analysis of the magnetic field.

\section{References}

(1) Higuchi, T., Applied Mechanical Engineering, (in Japanese), (1986), p. 92.

(2) Yabuuchi, K., Inoue, M., Akishita, S., Murakami, C. and Okamoto, O., A Compact Magnetic Bearing for Gimballed Momentum Wheel, Proc. 17 th Aerosp. Mech. Symp., (1983-5).

( 3 ) Nonami, K., Study of Machine,(in Japanese), 399(1987), p. 983.

(4) Humphris, R. R., Kelm, R. D., Lewis, D. W. and Allaire, P. E., Effect of Control Algorithms on Magnetic Journal Bearing Properties, J. Eng. Gas Turbine \& Power, Vol. 108, No. 4(1986), p. 624.

(5) Fukada, S., Kouya, Y. and Tamura, H., Dynamics of Active Magnetic Bearings, Trans. Jpn. Soc. Mech. Eng., (in Japanese), Vol. 53, No. 490, C (1987), p. 1201.

(6) Nakata, T. and Takahashi, N., Finite Element Method of Electrical Engineering, (in Japanese), (1983), Morikita Publishing Company, Tokyo. 